\title{
INVESTIGACIÓN
}

Recibido: 28/03/2019 --- Aceptado: 28/06/2019 --- Publicado: 15/09/2020

\section{FINANCIAMIENTO DE LAS CAMPAÑAS POLÍTICAS DE 2018 EN MÉXICO: DESIGUALDAD DE GÉNERO}

\author{
Financing of 2018 political campaigns in Mexico: gender inequality
}

Angélica Mendieta Ramírez: Benemérita Universidad Autónoma de Puebla. México angelica.mendietaram@correo.buap.mx

\section{RESUMEN}

En México existe una desigualdad de género entre hombres y mujeres que puede llevarse incluso al ámbito político: durante las campañas electorales para elegir a los 128 curules del senado y 500 diputados federales de las elecciones de 2018, los partidos invirtieron un 30 por ciento a las candidatas mujeres; mientras que los candidatos hombres recibieron un 70 por ciento del financiamiento. Mediante una metodología de investigación cuantitativa, se analizaron los reportes de financiamiento del Instituto Nacional Electoral y se demuestra la inequidad de género en las campañas políticas. Se propone realizar leyes y políticas compensatorias con perspectiva de género para garantizar candidaturas paritarias para las campañas políticas de las elecciones de 2021.

PALABRAS CLAVE: financiamiento - campañas políticas - desigualdad de género

\section{ABSTRACT}

In Mexico, there is a gener inequality between men and woman that can even take the political sphere: during electoral campaigns to elect the 128 seats in the senate and 500 federal deputies in the 2018 elections, the parties invested 30 percent in the elections. Female candidates; while the male candidates received 70 percent of the funding. Using a quantitative research methodology, analyze the financing reports of the National Electoral Institute and demonstrate gender inequality in political policies. It is proposed to carry out compensatory laws and policies with a gender perspective for joint candidates for the political campaigns of the 2021 elections.

KEY WORDS: financing - political campaigns - gender inequality 


\section{FINANCIAMENTO DAS CAMPANHAS POLÍTICAS DE 2018 NO MÉXICO: DESIGUALDADE DE GÊNERO}

\section{RESUMO}

No México existe uma desigualdade de gênero entre homens e mulheres que pode ser levado inclusive até o âmbito político: durante as campanhas eleitorais para escolher aos 128 lugares do senado e 500 deputados federais nas eleições de 2018, os partidos investiram 30 por cento nas candidatas mulheres; enquanto que os candidatos homens receberam 70 por cento do financiamento. Através de uma metodologia de pesquisa quantitativa, foram analisados os reportes de financiamento do Instituto Nacional Eleitoral e se demonstra a iniquidade de gênero nas campanhas políticas. Se pretende realizar leis e políticas compensatórias com perspectiva de género para garantir candidaturas igualitárias para as campanhas políticas das eleições de 2021.

PALAVRAS CHAVE: financiamento - campanhas políticas - desigualdade de gênero

\section{Como citar el artículo:}

Mendieta Ramírez, A. (2020). Financiamiento de las campañas políticas de 2018 en México: desigualdad de género. [Financing of 2018 political campaigns in Mexico: gender inequality]. Vivat Academia. Revista de Comunicación, 152, 101-115. doi: http:/ / doi.org/10.15178/va.2020.152.101-115 Recuperado de:

http://www.vivatacademia.net/index.php/vivat/article/view/1271

\section{INTRODUCCIÓN}

Las campañas electorales tienen la función de dar a conocer a los ciudadanos las propuestas de campaña y las promesas que cumplirán los candidatos y candidatas cuando logren llegar al poder. En México se ha logrado avanzar favorablemente en el reconocimiento de los derechos políticos de ambos géneros, sin embargo todavía prevalecen ejemplos de violencia política hacia las mujeres, discriminación y desigualdad en la inversión que realizan los partidos políticos hacia las candidatas.

Hasta antes de la Reforma Político Electoral de 2014, los partidos políticos no eran sujetos obligados dentro del régimen de transparencia y acceso a la información pública gubernamental, la información que se podía obtener acerca de la campañas electorales y el ejercicio de sus finanzas sólo se conocía por medio de los institutos electorales de las entidades de la República; era muy complicado hacer un análisis del dinero utilizado en las campañas electorales.

Pero poco a poco se avanzó en la democratización y transparencia. Las elecciones de 2018 fueron históricas; porque se logró la tercera alternancia electoral en el 
gobierno de la República, luego de dos décadas iniciales y después de 4 elecciones presidenciales. En el año 2000 después de 70 años de hegemonía del Partido Revolucionario Institucional (PRI), ocurrió la primera alternancia. Ganó Vicente Fox Quesada del Partido Acción Nacional (PAN), posteriormente volvió a ganar el PRI en el 2012 con Enrique Peña Nieto y en el 2018, el electorado se decantó por un gobierno de izquierda con el presidente Andrés Manuel López Obrador del Partido Movimiento de Regeneración Nacional (MORENA).

Durante la jornada electoral de 2018, los partidos políticos y candidatos independientes reportaron haber recibido en conjunto 5 mil 334 millones de pesos, además, al final de la elección, el Instituto Nacional Electoral (INE), detectó 184. 1 millones de pesos de gasto que no fue reportado, que representó el 3.5 por ciento del gasto del que sí rindieron cuentas los partidos políticos. En estas elecciones de 2018, se eligió al presidente de la República, 500 diputados federales y 128 senadores, 8 gobernadores, el titular de la jefatura de gobierno de la Ciudad de México, 27 congresos locales y mil 601 alcaldías (Murayama, 2019 p.15).

Esto ha producido una amplia manifestación democrática en la reivindicación de los derechos políticos de las minorías y buscado la equidad de hombres y mujeres en el acceso al poder. Pero, todavía los partidos políticos adoptan una visión machista y androcéntrica al postular a mayores hombres con posibilidades de ganar las elecciones y apuestan menos por las mujeres.

La esfera pública ha sido un ámbito en el que históricamente han tenido preeminencia los hombres. Ellos han sido los líderes, los candidatos y los electos mayoritariamente. En el informe: Ojos que no ven. Cobertura mediática y género en las elecciones Latinoamericanas, realizado en 2011 por ONU MUJERES, ${ }^{1}$ se visibilizó el problema de la inequidad mediática, sin embargo, tuvo poco eco en las legislaciones de América Latina y los partidos políticos no han adoptado una postura al respecto. Continúa la poca distribución financiera hacia las campañas de mujeres en nuestro país, así como en la distribución de pautas publicitarias.

Esta situación no es exclusiva de México, en Estados Unidos, antes de las elecciones intermedias de 2018, solo había 23 mujeres entre los 100 senadores y 84 entre los 435 congresistas del país más poderoso del mundo. ${ }^{2}$ Por tanto, la problemática de la inequidad mediática no sólo es una característica de nuestro país, un estudio realizado por la Oficina Federal de Comunicaciones de Suiza (OFCOM), la Oficina Federal de la Comisión de Asuntos de la Mujer y emisora pública suiza (SRG SSR), publicado en 2015, concluyó que si bien las mujeres representaban el 34,5

\footnotetext{
1 Véase: Llanos Beatriz (2011), Ojos que no ven: cobertura mediática y género en las elecciones latinoamericanas, ONU MUJERES, IDEA, consultado el 12 de mayo de 2019, disponible en línea: https:// www.idea.int/publications/catalogue/ojos-que-no-ven-cobertura-medi\%C3\%A1tica-yg\%C3\%A9nero-en-las-eleccciones

2 Véase: Amanda Mars y Pablo Guimón (2018), Elecciones en EE UU: mujeres al poder, El País, 28 de octubre de 2019, consultado el 29 de mayo de 2019. Disponible en línea: https://elpais.com/internacional/2018/10/26/actualidad/1540574302_751574.html
} 
por ciento de las candidaturas al Consejo Nacional Suizo (la cámara baja de la Asamblea Federal), en contraste sólo aparecieron en un 24 por ciento en las historia de audio y video de las campañas televisivas, un 23.5 por ciento en los relatos e información publicada en medios impresos y en línea; así como en un 25 por ciento de las fotos publicadas en revistas y periódicos. Lo cual resulta preocupante si se comparan las cifras de 2003 y 2015 en Suiza, porque prácticamente no se ha avanzado en la cobertura mediática más allá del 25 por ciento (CE, 2017, p. 10).

Esta inequidad puedes ser comprobada por las investigaciones de Vargas \& Palazuelos (2019), Elizondo (2017) y; Font y Matías (2019). La relación asimétrica entre hombres y mujeres también permea el ámbito de la política, porque se reproducen los estereotipos, roles, prejuicios y toda serie de discriminación y trato desigual; producto de la cultura e idiosincrasia (Estrada et. al., 2016). Existen múltiples factores que acotan y limitan la participación de las mujeres, por ejemplo.

Pese a ello, si hay avances. La Reforma Política, promulgada en 2014 por el ex presidente Enrique Peña Nieto, permitió alcanzar la igualdad sustantiva entre hombres y mujeres. Se decretó que los partidos políticos estaban obligados a postular en igual porcentaje a hombres y mujeres. El 50 por ciento de las candidaturas desde ese año, son para hombres y 50 por ciento para mujeres.

La agenda de género en materia electoral inició en la desde la década de los cincuentas, después de que se logró en México que las mujeres pudieran salir a votar las elecciones del 3 de julio de 1955. También se logró contar con mujeres como representantes políticos en varios países. Chile contó con mujeres en el poder desde 1946, como representantes políticos; Cuba en 1960; México, Bolivia, Costa Rica, República Dominicana y Perú, iniciaron en la década de los setentas luchas por participar en la política; mediante organizaciones y grupos de presión (Zaremberg, 2008, p. 29).

El avance de la participación política de las mujeres en México, ha sido de manera paulatina, En 1993, en la fracción 3 del artículo 175, el Código Federal de Instituciones y Procedimientos Electorales (COFIPE) solamente recomendaba que los partidos promovieran la mayor participación de las mujeres a través de la postulación de los cargos de elección, es decir; no existía un compromiso democrático desde el Estado para fomentar la inclusión de mujeres en la política. Se denominó pro-cuotas, porque estaba desvinculada del interés real de que las mujeres pudieran acceder al poder (Caminotti \& Freidenberg, 2016, p. 124). Posteriormente en la siguiente reforma del COFIPE en1996 se incluyó la recomendación de que en las candidaturas no se excediera 70 por ciento de postulaciones de un mismo género, tanto en la representación proporcional como en la mayoría relativa. ${ }^{3}$

\footnotetext{
${ }^{3}$ Empero no existía una reglamentación clara sobre las sanciones en caso de incumplir dichas cuotas, veáse: Freidenberg, (2017), donde describe el contexto histórico de este proceso.
} 
Fue hasta el 2002 que se estableció en el COFIPE, en el artículo 175, inciso A, un sistema de cuotas en el cual las postulaciones de candidatos a diputados y senadores no podrían ser mayor al 70 por ciento. Por lo cual, las mujeres ganaron un 30 por ciento de representación política en la legislatura. En 2006, la legislatura federal compuesta por 500 curules estaba distribuida así: 212 eran legisladoras (42.4 por ciento), en tanto que 288 eran hombres (57.6 por ciento). ${ }^{4}$ Para 2016, en el senado ocurría lo mismo, de los 128 espacios del senado, 81 estaban compuestos por hombres y 47 por mujeres, es decir, respectivamente la representación era de 64.6 y 35.4 por ciento. Posteriormente en el 2008, la reforma en el artículo 219 del COFIPE establecía que el porcentaje de candidaturas propietarias debía ser de al menos el 40 por ciento, además de incluir un 2 por ciento del dinero otorgado a los partidos políticos, para promover cursos y talleres de liderazgo político. Fueron varias las acciones afirmativas para insertar a las mujeres en la política, ${ }^{5}$ pero no sólo dentro de este ámbito, sino también a partir de una mayor inclusión educativa y de acceso al mercado laboral.

Hasta la Reforma Política de 2014, ${ }^{6}$ fue cuando se estableció el concepto de paridad de género en las candidaturas de los partidos políticos que se vieron obligados a incluir a las féminas en las postulaciones a cargos de elección popular. Sobre la participación política de las mujeres hay múltiples estudios, Guadarrama (2015), Archenti (2011), y otros. A nivel mundial, el 78 por ciento de las candidaturas corresponde a los hombres, hay una sobre representación masculina en todo el mundo sobre lo cual están trabajando los gobiernos para disminuir esta brecha de género (IDEA, 2016).

En torno a la violencia política hacia las mujeres también se han documentado múltiples experiencias de nuestro país: Granados (2014), Moscoso (2012), Durán, et. al. (2014), siendo la inequidad en las campañas políticas considerada como una violencia de género.

Con base en el manual de IDEA Internacional y ONU Mujeres en Mirando con lentes de género la cobertura electoral. Manual de monitoreo de medios (Llanos \& Nina, 2011), encontramos que el uso discrecional de los recursos públicos en las campañas electorales requiere mecanismos de control, tanto hacia los partidos políticos en las dirigencias nacionales; como también en las finanzas y transparencia

\footnotetext{
4 Véase: Jiménez Horacio y Alberto Morales (2016), "Limitan poder de mujeres en congreso", Periódico El Universal, consultado el 23 de febrero de 2018, disponible en línea: http://www.eluniversal.com.mx/articulo/nacion/politica/2016/03/8/limitan-poder-de-mujeres-encongreso

${ }^{5}$ Dentro de las acciones afirmativas destaca el lenguaje incluyente, así como todas aquellas medidas correctivas tendientes a aumentar la participación de las mujeres en la actividad electoral, como la capacitación electoral y la posibilidad de insertarse en los espacios para la toma de decisiones.

6 El 10 de febrero de 2014 se aprobó la Reforma Constitucional al artículo 41, estableciendo con ello que los partidos políticos deben garantizar la paridad de géneros en las candidaturas a las legislatura federal y las legislaturas locales.
} 
en los recursos públicos. Por eso han surgido los observatorios vinculados con las universidades y con las organizaciones de la sociedad civil.

Por ejemplo, en Italia, desde 2012, los medios italianos están obligados por ley ("par condicio di genere") a proporcionar una representación equilibrada de mujeres y hombres en la cobertura de sus campañas electorales y políticas, que se realizan también sus programas televisivos y de radio. Se pidió a la autoridad reguladora italiana (AGCOM) que publicara datos sobre el Presencia de mujeres políticas en programas políticos de radio y televisión, pero no existe ningún informe hasta la fecha. Una de las razones por las que adelantaron algunas mujeres. Grupos es que dicho informe sería un desafío, considerando que los candidatos son principalmente hombres (CE, 207). Sin embargo, en Europa también las mujeres están sujetas a discriminación y existe mayor visibilidad de los candidatos hombres en los noticieros y programas.

En América Latina, todavía la legislación es muy incipiente; porque los partidos políticos han crecido sin mayor control. Rial (2015), sostiene que cada vez es más difícil fiscalizar y controlar el financiamiento, porque ante las nuevas formas de promocionar las candidaturas en WhatsApp o en redes sociales; existen serias limitaciones para garantizar la equidad de las contiendas.

Solo 41 países tienen más del 30 por ciento de mujeres en sus parlamentarios aproximadamente una quinta parte de todos los países - y solo 12 por ciento a nivel mundial, tienen 40 por ciento o más de mujeres en sus parlamentos. Es decir, todavía subsiste una distribución inequitativa en la representación política (IDEA, 2016).

Derivado de los argumentos anteriores, desde el ámbito de la comunicación política el financiamiento que reciben los partidos políticos para hacer campaña determina en parte el éxito electoral. Se parte de la hipótesis de que el financiamiento es un elemento que promueve la compra de productos comunicativos y por tanto, permite una mayor posibilidad de triunfo de los candidatos en las contiendas electorales; para el caso de las mujeres candidatas, contar con menos presupuesto está incidiendo en probabilidad de éxito electoral. Por lo cual debe promoverse una agenda de equidad de género para que los candidatos y las candidatas puedan disminuir la brecha de desigualdad financiera que tienen para garantizar una mayor calidad democrática.

\section{OBJETIVOS}

El objetivo de esta investigación es analizar los recursos públicos que recibieron los partidos políticos durante las elecciones de 2018, con base en los informes financieros del Instituto Nacional Electoral con el fin de comprobar la distribución en las campañas electorales.

\section{METODOLOGÍA}


En esta investigación se utilizó el análisis cuantitativo, para visualizar a partir de las variables de género: candidatos y candidatas a ocupar un cargo dentro de los 500 diputados federales y 128 senadores. Se eligió a estos dos tipos de representantes políticos porque durante las elecciones de 2018, fueron las más importantes además de la presidencia de la Republica; que solo conto con candidatos varones: José Antonio Meade Kuribeña por la Coalición Todos por México, encabezada por el PRI, Ricardo Anaya Cortés por la Coalición Pro México al frente, encabezada por el PAN y el candidato independiente Jaime Rodríguez Calderón. Al no existir una candidata a la presidencia fue imposible hacer el comparativo a nivel nacional de los recursos erogados por los partidos políticos para la Presidencia de la República en México.

Para obtener la información se hizo una solicitud de transparencia al Instituto Nacional Electoral, como sujeto obligado para atender las solicitudes de información. En un ejercicio de rendición de cuentas, se solicitó el Informe He for She, Reporte de la Distribución del Ingreso y Gasto en las Campañas Electorales de 2018, por partido político (MOCESP, 2018).

Contar con información y datos del financiamiento de los partidos políticos es un asunto reciente. Hasta antes de la Ley de Transparencia y Acceso a la Información Pública, aprobada en el año de abril de 2016; permite que los partidos políticos sean sujetos obligados.

En cuanto a la transparencia, el primer cambio en el sistema de partidos se dio en 1976 cuando se aprobó otorgar a los partidos políticos financiamiento público, por parte del Estado. Aunque poco se construyó en términos de control hacia este dinero público. En 1977 se expidió la Ley General de Organizaciones Políticas y Procesos Electorales (LOOPE) y que llevó a la creación del Instituto Nacional Electoral en 1990. Antes, en el año de 1977 con la reforma en el artículo sexto de la Constitución Mexicana que se reconoció el derecho de acceso a la información pública gubernamental; pero se cristalizó en 1993 con el Código Federal de Procedimientos Electorales (COFIPE), en el artículo quinto.

En 1996 se modificó las reglas del juego para acceder al financiamiento de los partidos, condicionando el financiamiento privado a los institutos políticos, como la vía para garantizar la equidad en las contiendas electorales. Asimismo, en este mismo año; después de las elecciones de 1994 cuando ganó Ernesto Zedillo Ponce de León emanado del PRI, como presidente de la República, se extendieron las facultades de fiscalización del Instituto Federal Electoral que fue creado en 1990.

Más adelante en 2014 se impulsó la Reforma Político Electoral que constituyó un rediseño en el régimen electoral, en principio se modificó el Instituto Federal Electoral, que pasó a ser el Instituto Nacional Electoral (INE), asimismo construyó los cambios en el COFIPE para dar pie a la Ley General de Instituciones y Procedimientos Electorales (LEGIPE), promulgada el 23 de mayo de 2014 y modificada en el 27 de enero de 2017; donde se contemplan las obligaciones más amplias para los partidos políticos. De manera paralela, otra institución pilar de la 
democracia fue modificada; el Instituto Federal de Acceso a la Información Pública Gubernamental (IFAI), modificó su nombre a Instituto Nacional de Transparencia, Acceso a la Información y Protección de Datos Personales (INAI), como parte de las modificaciones a la Ley General de Transparencia y Acceso a la Información Pública, promulgadas por el presidente Enrique Peña Nieto en 2015; con esta transformación, al INAI se le otorgaron facultades para vigilar a los partidos políticos y sindicatos.

\section{RESULTADOS}

Derivado de las leyes anteriormente expuestas, así como de la democratización de los partidos políticos que tuvieron que abrir la información sobre el financiamiento que se recibe, a continuación se muestra el análisis de los ingresos que recibieron las campañas políticas durante la elección de 2018 para los 128 candidatos y candidatas al senado y un espacio de representación en la Cámara de Diputados compuesta por 500 curules.

Chacón (2011), sostiene que si bien es cierto que las campañas han tenido una amplia vulnerabilidad en la transparencia y uso de recursos ilícitos derivados de donaciones, así como uso de los recursos públicos; la legislación en nuestro país es sólida y busca alejar a los políticos de las fuentes de financiamiento que no corresponden a un país democrático.

Tabla 1. Distribución del ingreso de las campañas.

\begin{tabular}{|c|c|c|c|c|c|c|}
\hline \multirow[b]{2}{*}{ Rubro } & \multicolumn{2}{|c|}{ Mujeres } & \multicolumn{2}{|c|}{ Hombres } & \multicolumn{2}{|c|}{ Total Recurso } \\
\hline & Monto & $\%$ & Monto & $\%$ & Monto $\bar{z}$ & $\%$ \\
\hline Transferencias de recurses locales & $\$ 508,230,687.39$ & $38.31 \%$ & $\$ 818,496,853.5$ & $61.69 \%$ & $\$ 1,326,727,540.89$ & $100.00 \%$ \\
\hline Aportaciones de simpatizantes & $79,842,159.7$ & 35.05 & $147,955,540.14$ & 64.95 & $227,797,699.84$ & 100.00 \\
\hline Transferencias de recurses federales & $28,835,127.34$ & 32.48 & $59,956,283.65$ & 67.52 & $88,791,410.99$ & 100.00 \\
\hline Aportaciones del candidato & $27,616,074.8$ & 36.61 & $47,823,101.76$ & 63.39 & $75,439,176.56$ & 100.00 \\
\hline Ingresos por transferencias en especie & $24,656,417,4$ & 41.76 & $34,383,733.01$ & 58.24 & $59,040,150,41$ & 100.00 \\
\hline Aportaciones de militantes & $21,076,312.89$ & 38.32 & $33,931,127.23$ & 61.68 & $55,007,440.12$ & 100.00 \\
\hline Transferencias de Candidatos R.P. Locales & $107,188.03$ & 49.20 & $110,667.77$ & 50.80 & $217,855.8$ & 100.00 \\
\hline Transferencias de candidatos R.P. federales & $97,749.92$ & 54.53 & $81,514.86$ & 45.47 & $179,264.78$ & 100.00 \\
\hline Otros ingresos & $16,423.11$ & 15.35 & $90,561.16$ & 84.65 & $106,984.27$ & 100.00 \\
\hline Rendimientos financieros, fondos y fideicomisos & 199.53 & 0.21 & $95,261.39$ & 99.79 & $95,460.92$ & 100.00 \\
\hline Autofinanciamiento & $9,175.32$ & 85.15 & $1,600.11$ & 14.85 & $10,775,43$ & 100.00 \\
\hline Total general & $690,487,515,43$ & 37.66 & $1,142,926,244.58$ & 62.34 & $1,833,413,760.01$ & 100.00 \\
\hline
\end{tabular}

Fuente: Instituto Nacional Electoral 2020.

Como puede apreciarse en la tabla 1, sobre la distribución del ingreso de las campañas electorales de las y los candidatos a diputados federales y senadores, tuvo un monto total de 1 billón, 833 mil 413 mil 760 pesos; de los cuales se destinó un total de 690 millones, 487 mil 515.43 en las campañas de las mujeres candidatas; mientras que para las campañas de los hombres candidatos se destinó un total de un mil 
millones, 142 mil 926 millones con 244 pesos. Esto significa que las mujeres recibieron un 37.66 por ciento del financiamiento, mientras que los hombres recibieron el 62.34 por ciento.

Casar y Ugalde (2018), sostienen que existen ciertas zonas vulnerables al uso de recursos de dudosa procedencia, con influencia de otros actores que buscan incidir en la política local. A nivel municipal es más fácil que se presenten este tipo de casos; dado que la normatividad carece de esquemas que vayan hacia la vigilancia cercana o sobre la influencia que tienen los caciques y actores locales que utilizan financiamiento privado y desvió de recursos públicos en menor escala. Pero actualmente a través de las nuevas tecnologías se está dando un mayor seguimiento a las acciones de los partidos políticos, porque existen fotos y videos que inundan la red sobre las campañas electorales y ejercicios de observación electoral.

El Código Federal de Instituciones y Procedimientos electorales, promulgado en 1990 no consideraba como una obligación permitir la práctica de autorías a los institutos políticos por parte de la autoridad electoral; sino hasta 1996 que se reformó la ley para exigir la rendición de cuentas. El antecedente más importante de mecanismos para transparentar a los partidos políticos fue en 1993 con el COFIPE, que establecía en el artículo 5, la posibilidad de impulsar vigilancia en el proceso electoral y en los partidos políticos; así como el establecimiento jurídico de obligar a los partidos políticos a rendir informes sobre sus ingresos y gastos ante la autoridad electoral

En la distribución de los recursos, los partidos políticos sí muestran diferencias en la inversión a candidatos y candidatas, como se muestra en el cuadro 2. Mientras que el Partido Acción Nacional registró el ingreso del 24.72 por ciento para las candidaturas de las mujeres candidatas, Morena logró un porcentaje del 49.49 por ciento. Muy cercano al 50 por ciento.

Tabla 2. Distribución del ingreso por partido político. 
Mendieta Ramírez, A.

Financiamiento de las campañas políticas de 2018 en México: desigualdad de género

\begin{tabular}{|c|c|c|c|c|c|c|}
\hline \multirow[b]{2}{*}{ Partido/Coalición } & \multicolumn{2}{|c|}{ Mujeres } & \multicolumn{2}{|c|}{ Hombres } & \multicolumn{2}{|c|}{ Total general } \\
\hline & Total Ingreso & $\%$ & Total Ingreso & $\%$ & Total Ingreso & $\%$ \\
\hline Partido Acción Nacional & $41,064,803.57$ & 24.72 & $125,070,736.8$ & 75.28 & $166,135,540.37$ & 100.00 \\
\hline Partido Revolucionario Institucional & $127,017,183.11$ & 37.21 & $214,331,798.71$ & 62.79 & $341,348,981.82$ & 100.00 \\
\hline Partido de la Revolución Democrática. & $32,019,602.91$ & 39.84 & $48,346,980.45$ & 60.16 & $80,366,583.36$ & 100.00 \\
\hline Partido Verde Ecologista de México & $62,153,398.96$ & 37.98 & $101,496,284.62$ & 62.02 & $163,649,683.58$ & 100.00 \\
\hline Partido del Trabajo & $7,013,057,9$ & 36.68 & $12,104,556.78$ & 63.32 & $19,117,614.68$ & 100.00 \\
\hline Movimiento Ciudadano & $31,210,459.29$ & 37.84 & $51,273,844.39$ & 62.16 & $82,484,303.68$ & 100.00 \\
\hline Nueva Alianza & $44,863,493.91$ & 40.89 & $64,853,064.43$ & 59.11 & $109,716,558.34$ & 100.00 \\
\hline Morena & $27,780,763.93$ & 49.49 & $28,357,338.02$ & 50.51 & $56,138,101.95$ & 100.00 \\
\hline Encuentro Social & $11,104,620.27$ & 43.88 & $14,203,190.99$ & 56.12 & $25,307,811.26$ & 100.00 \\
\hline Juntos Haremos Historia & $87,631,597.42$ & 42.33 & $119,385,238.22$ & 57.67 & $207,016,835.64$ & 100.00 \\
\hline Por México al Frente & $157,204,875.31$ & 38.38 & $252,400,581.93$ & 61.62 & $409,605,457.24$ & 100.00 \\
\hline Todos por México & $39,319,493.7$ & 35.72 & $70,759,145.63$ & 64.28 & $110,078,639.33$ & 100.00 \\
\hline Partidos Locales & $22,104,165.15$ & 35.40 & $40,343,483.61$ & 64.60 & $62,447,648.76$ & 100.00 \\
\hline Total general & $690,487,515.43$ & 37.66 & $1,142,926,244.58$ & 62.34 & $1,833,413,760.01$ & 100.00 \\
\hline
\end{tabular}

Fuente: Instituto Nacional Electoral 2020.

El tema de los recursos económicos destinados a las campañas influye en los resultados por la posibilidad que tienen los candidatos de incidir en la opinión pública; por medio de la exposición en los productos comunicativos; pero también existen limitaciones por parte de las mujeres en cuanto a tiempo y posibilidad de participar en la política. IDEA (2016), encontró que las mujeres se encuentran en franca desventaja cuando son candidatas porque sus redes de contactos y simpatizantes son menores a las de los hombres.

Las mujeres deben trabajar para ganar adeptos, porque la exposición mediática es menor; pero también sus redes de contactos y simpatizantes suelen ser más limitadas.

Tabla 3. Distribución del gasto 


\begin{tabular}{|c|c|c|c|c|c|c|}
\hline \multirow[b]{2}{*}{ Rubro } & \multicolumn{2}{|c|}{ Mujeres } & \multicolumn{2}{|c|}{ Hombres } & \multicolumn{2}{|c|}{ Total Recurso } \\
\hline & Monto & $\%$ & Monto & $\%$ & Monto $=$ & $\%$ \\
\hline Propaganda utilitaria & $\$ 224,574,606.91$ & $40.89 \%$ & $\$ 324,683,053.34$ & $59.11 \%$ & $\$ 549,257,660.25$ & $100.00 \%$ \\
\hline Operatives de la campaña & $185,737,486.91$ & 37.17 & $313,903,475.88$ & 62.83 & $499,640,962.79$ & 100.00 \\
\hline Propaganda & $197,028,928.24$ & 39.51 & $301,693,575.13$ & 60.49 & $498,722,503.37$ & 100.00 \\
\hline Propaganda en vía pública & $34,668,442.95$ & 26.50 & $96,132,960.92$ & 73.50 & $130,801,423.87$ & 100.00 \\
\hline Propaganda exhibida en páginas de internet & $16,300,280.38$ & 27.83 & $42,271,338,40$ & 72.17 & $58,571,618.78$ & 100.00 \\
\hline Propaganda en diarios, revistas y otros medios impresos & $7,840,890.88$ & 28.76 & $19,418,590.45$ & 71.24 & $27,259,471.33$ & 100.00 \\
\hline Producción de los mensajes para radio y T.V. & $3,152,938.52$ & 36.69 & $5,441,164.78$ & 63.31 & $8,594,103.30$ & 100.00 \\
\hline Propaganda exhibida en salas de cine & $1,743,691,80$ & 37.56 & $2,899,177.10$ & 62.44 & $4,642,868.90$ & 100.00 \\
\hline Financieros & $609,215.13$ & 47.73 & $667,098.43$ & 52.27 & $1,276,313.56$ & 100.00 \\
\hline Total general & $671,656,471.72$ & 37.76 & $1,107,110,454.43$ & 62.24 & $1,778,766,926.15$ & 100.00 \\
\hline
\end{tabular}

Fuente: Instituto Nacional Electoral 2020.

En la tabla 3, puede apreciarse que por ejemplo en la propaganda y producción de mensajes, las candidatas mujeres recibieron menos del 40 por ciento, mientras que los candidatos hombres, recibieron del 60 al 70 por ciento de los recursos. Un rubro que durante las elecciones de 2018 fue muy importante, es la propaganda en páginas web; donde las mujeres a cargos de elección popular recibieron solamente el 27.83 por ciento, mientras que los hombres tuvieron un monto de 72.17 por ciento.

Las tablas 1, 2 y 3; muestran que los existe una distribución inequitativa en términos de inversión en las campañas electorales de los partidos políticos hacia los y las candidatas.

\section{CONCLUSIONES}

Como se puede apreciar en los resultados obtenidos, las campañas electorales en México tienen un fuerte sesgo cultural, donde se reproducen los estereotipos en los productos comunicativos que se difunden para atraer a los electores. En ese sentido, las mujeres se encuentran en desventaja estructural e institucional para llevar a cabo una campaña en igualdad de condiciones. Esto es una limitante en términos democráticos, que altera la integridad electoral para las próximas elecciones de 2021 donde se votará nuevamente por 500 diputados federales y diputados locales de 30 entidades del país, así como 15 gubernaturas.

Pero también se encontró que entre los partidos políticos existen diferencias en su vocación de género. El partido que registró casi el 50 por ciento de ingresos a las candidaturas de las mujeres fue Morena, mientras que el más inequitativo fue el PAN, durante las elecciones de 2018. En ese sentido, Gottlieb (2016), encontró en Malí, dentro del continente africano que los ciudadanos están dispuestos a participar cuando existe más información sobre las acciones de los representantes o legisladores. La formación cívica es clave para garantizar también contar con más 
adeptos dentro de los partidos políticos y hacer que los militantes, así como simpatizantes puedan tener más información sobre las metas y los objetivos

Norris \& Nai (2017), sostiene que las elecciones requieren contar con una fuerte vigilancia en términos de transparencia del procedimiento electoral; pero también en cuanto a las finanzas de los partidos políticos. El 25 de junio de 2019 el INE sancionó al partido Movimiento de Regeneración Nacional (MORENA), por haber incumplido sus obligaciones en materia de transparencia. El monto de la sanción correspondió a tres multas de 80 mil 600 pesos que, sumadas, ascienden a 241 mil 800 pesos; que fueron descontadas del total de sus prerrogativas para el meses de junio de 2019 que corresponden a $\$ 115,584,056.00$ (ciento quince millones quinientos ochenta y cuatro mil cincuenta y seis pesos 00/100 M.N.) ${ }^{7}$

Es importante resaltar que Morena ha sido sancionado en 12 ocasiones durante 2019 por omisiones que ocurrieron en 2018, relacionadas con las obligaciones que estipula la ley de transparencia aplicable a los partidos políticos. Las otras sanciones se debieron a que no se publica el tabulador de sueldos que perciben los funcionarios del instituto político. Tampoco se publican los gastos de representación y viáticos, como debería mostrarse. Otro faltante es que no se informa sobre los contratos de bienes y servicios o no se publican mecanismos y procesos de selección de candidatos (Hernández, 2019). En el balance de las elecciones de 2018, se dio a conocer que las sanciones a los partidos políticos por sanciones en términos de fiscalización fueron de 870 millones de pesos. ${ }^{8}$

Si bien es cierto que la ley debe controlar a los partidos políticos en términos del financiamiento que reciben, también es preciso establecer mecanismos compensatorios para garantizar la equidad de género en términos de financiamiento. Esto puede lograrse a partir de la transparencia y rendición de cuentas hacia los partidos políticos por medio de organizaciones de la sociedad civil. La contraloría social que se produce con la incidencia de organizaciones que vigilan el cumplimiento de los derechos sociales y políticos, puede tener buenos resultados. Además, del tema financiero, la brecha de desigualdad se muestra en la poca incidencia de las mujeres en la vida pública, en la creación de iniciativas y también en la marginalidad que existe en mujeres que pertenezcan a las minorías: indígenas y pertenecientes a distintas etnias donde apenas han logrado el derecho al voto (Dahlerup, 2010).

Ante este panorama, es preciso construir una mayor cultura política en los representantes partidistas, legisladores e integrantes de los partidos políticos; para lograr incidir en la toma de decisiones y disminuir la brecha presupuestal que existe

\footnotetext{
7 Véase: Expediente: UT/SCG/Q/INAI/CG/302/2018, correspondiente al acta de consejo del 25 de junio de dos mil diecinueve.

8 Véase: Maritza Pérez, Rolando Ramos y Elizabeth Albarrán (2019), INE multa a partidos con más de 500 mdp, El Economista, 6 de noviembre de 2019. Disponible en línea: https:// www.eleconomista.com.mx/politica/INE-multa-a-partidos-con-mas-de-500-mdp-20191106$\underline{0107 . h t m l}$
} 
en la inversión que se realiza a los candidatos y candidatas. Si se logró recientemente en 2014, la paridad de género en las candidaturas, ahora es necesario garantizar también la paridad financiera de los y las candidatas para tener una mejor calidad en la democracia de nuestro país.

IDEA (2016), sostiene que los partidos políticos pueden beneficiarse de las políticas de equidad de género; no sólo en la distribución de las candidaturas entre hombres y mujeres, sino también en la equidad financiera. Acciones de reivindicación de género producen producir mayor participación electoral, legitimidad en las elecciones y por supuesto una cultura de democrática incluyente.

A partir de este análisis y estudio, se abren las posibilidades para que otros países también puedan reflexionar en torno a este fenómeno anti democrático que persiste y que puede tener un impacto en las siguientes elecciones, para México en el 2021; pero también para otros países de América Latina.

\section{REFERENCIAS}

Archenti, N. (2011). La paridad política en América Latina y el Caribe. Percepciones y opiniones de los líderes de la región. Serie Mujer y Desarrollo. División Asuntos de Género, Santiago de Chile: CEPAL.

Caminotti, M. y Freidenberg, F. (2016). Federalismo electoral, fortaleza de las cuotas de género y representación política de las mujeres en los ámbitos subnacionales de Argentina y México, en Revista Mexicana de Ciencias Políticas y Sociales, vol. 61 (228), 2016, pp. 121-141.

Casar, M.A. y Ugalde L. C. (2019). Dinero bajo la mesa. Financiamiento gasto ilegal de las campañas políticas en México, Ciudad de México: editorial Grijalbo.

CE (Council of Europe) (2017). Study on media coverage of elections with a specific focus on gender equality, Committee of experts on Media Pluralism and Transparency of Media Ownership (MSI-MED). Recuperado de https://rm.coe.int/study-gender\%20-equality-in-media-coverage-ofelections $/ 1680776164$

Dahlerup Drude (2016). Women's Political Representation, Nancy A Napoles (2016), The Wiley Blackwell Encyclopedia of Gender and Sexuality Studies, First Edition.

Durán, M. I., Campos-Romero, I. y Martínez-Pecino, R. (2014). Obstáculos en la comprensión de la violencia de género: Influencia del sexismo y la formación, Acción Psicológica, 11(2), pp. 97-106. 
Estrada J.L., Mendieta A. y González B. (2016). Perspectiva de género en México: análisis de los obstáculos y limitaciones, Opción, año 32, especial número 13, pp. 12-36.

Font, S. y Ponce, M. (2019). Desigualdad mediática: las senadoras en la cobertura de los medios uruguayos. Cuadernos.Info, (45), 113-128. Recuperado de https://doi.org/10.7764/cdi.45.1550.

Gottlieb, J. (2016). Greater Expectations: A Field Experiment to Improve Accountability in Mali, American Journal of Political Science, 60 (1): 143-57.

Guadarrama, G. J. (2015). Un lugar indefinido. El género en la agenda municipal. La construcción de la política nacional en el Estado de México, El Colegio Mexiquense, Estado de México, México.

Granados, O. (2014). “México: cinco mujeres mueren al día en el país víctimas de la violencia", El País. $\quad$ Recuperado de http://internacional.elpais.com/internacional/2014/11/18/actualidad/14163398 30_910235.html

Hernández, E. (2019),"Sanciona INE a Morena por negar información", Reforma, miércoles 26 de junio de 2019.

IDEA (International Institute for Democracy and Electoral Assistance) (2016), A Framework for Developing Gender Policies for Political Parties, Suecia.

Llanos, B. (2011), Ojos que no ven: cobertura mediática y género en las elecciones latinoamericanas, ONU MUJERES, IDEA, consultado el 12 de mayo de 2019. Recuperado de https://www.idea.int/publications/catalogue/ojos-que-no-vencobertura-medi\%C3\%A1tica-y-g\%C3\%A9nero-en-las-eleccciones

Llanos, B. y Nina, J. (2011). Mirando con lentes de género la cobertura electoral. Manual de monitoreo de medios (Looking at the electoral coverage with gender eyes. Media monitoring manual). Lima: Instituto Internacional para la Democracia y la Asistencia Electoral (IDEA Internacional) - ONU Mujeres, Nueva York, USA.

Moscoso, V., Pérez A. y Estrada M. de la L. (2012). Violencia contra las mujeres en el Estado de México, Comisión Mexicana de Defensa y Promoción de los Derechos Humanos, México.

MOCESP (2018). Monitoreo de Campañas y Estereotipos en los Spots Pautados 2018. Instituto Nacional Electoral [obtenido el 28 de noviembre de 2018, mediante solicitud de transparencia]. Ciudad de México, México.

Murayama, C. (2019). La democracia a prueba. Elecciones en la era de la posverdad, Ciudad de México: Ediciones Cal y Arena 
Rial, J. (2015). Medios y campañas electorales. Retos para la equidad, Revista Derecho Electoral, Tribunal Supremo de Elecciones, número 19, pp. 328-341.

Norris P. y Nai, A. (2017). Election watchdogs. Transparency, accountability and Integrity, Oxford, University Press, USA.

Norris, P. y Lovenduski, J. (1994). Gender and Party Politics ((Thousand Oaks, CA: SAGE Publications, 1994) Publications Ltd, 1994)

Zaremberg, G. (2008), Mujeres, votos y asistencia social en el México priísta y la argentina peronista, Facultad Latinoamericana de Ciencias Sociales, México.

\section{AUTORES:}

\section{Angélica Mendieta Ramírez}

Doctora en Sociología, Posdoctorado en Educación, Maestra en Ciencias Políticas, Licenciada en Derecho, Licenciada en Ciencias de la Comunicación, ha realizado tres estancias: en la University of Harvard (2004), en The City University of New York (2014), y en la Universidad Complutense de Madrid (2014). Es miembro del SNI nivel I. Responsable del Cuerpo Académico Consolidado: "Comunicación Política" Es autora de los conceptos: Electopartidismo y el Bucle de la Comunicación Política. Es autora y coautora de más de 35 libros y más de 25 artículos en revistas arbitradas e indexadas entre los que destacan: “Diseños de investigación. El coaching metodológico como estrategia", "La democracia en tiempos de incertidumbre", "Cultura política de las mujeres en el estado de Puebla". Es fundadora y Directora General de la Asociación Mundial de Investigadores, A.C. Actualmente es Directora de la Facultad de Ciencias de la Comunicación de la BUAP (2017-2021).

angelicamendietaramirez@gmail.com

Orcid ID: https:// orcid.org/0000-0001-9344-8653 